\title{
Can Satellite Remote Sensing be Applied in Geological Mapping in Tropics?
}

\author{
Janusz Magiera ${ }^{1, *}$ \\ ${ }^{1}$ AGH University of Science and Technology, Faculty of Geology, Geophysics and Environmental \\ Protection, A. Mickiewicza Av. 30, 30-059 Krakow, Poland
}

\begin{abstract}
Remote sensing (RS) techniques are based on spectral data registered by RS scanners as energy reflected from the Earth's surface or emitted by it. In "geological" RS the reflectance (or emittence) should come from rock or sediment. The problem in tropical and subtropical areas is a dense vegetation. Spectral response from the rocks and sediments is gathered only from the gaps among the trees and shrubs. Images of high resolution are appreciated here, therefore. New generation of satellites and scanners (Digital Globe WV2, WV3 and WV4) yield imagery of spatial resolution of $2 \mathrm{~m}$ and up to 16 spectral bands (WV3). Images acquired by Landsat (TM, ETM+, OLI) and Sentinel 2 have good spectral resolution too (6-12 bands in visible and infrared) and, despite lower spatial resolution (10-60 m of pixel size) are useful in extracting lithological information too. Lithological RS map may reveal good precision (down to a single rock or outcrop of a meter size). Supplemented with the analysis of Digital Elevation Model and high resolution ortophotomaps (Google Maps, Bing etc.) allows for quick and cheap mapping of unsurveyed areas. Key words - Remote Sensing, geological mapping, tropics.
\end{abstract}

\section{Peculiarities of geological Remote Sensing in tropics}

Remote Sensing techniques are based on spectral data registered by RS scanner as energy reflected from the Earth's surface or emitted by it. The data can be imaging or non-imaging. In both cases it is essential that the reflectance (or emittence) comes from the objects on the Earth surface which are in focus of our interest, i.e. rock, sediment, or, to a lesser extent, soil $[1,2]$. And here a problem arises in tropical, subtropical and other areas with dense vegetation: tree canopies form exceptionally effective obstacle for direct electromagnetic "communication" between geological object and scanner (the only exception is microwave or radar radiation which penetrates the vegetation cover or even top layers of soft sediments). Spectral information on the rocks and sediments can be gathered only from the gaps among the trees and shrubs and from clearings. Therefore, spatial resolution of the images is a crucial parameter: the smaller the pixel the more selective (precise) spectral information is. Two more factors control effectiveness of RS analysis: spectral and radiometric resolution. The former is defined by the number of spectral bands, the latter - by the number of bits of information per pixel (in both cases - the more the better). Temporal resolution (repeatability

* Corresponding author: magiera@geol.agh.edu.pl 
of imaging of a given area), which is essential for monitoring of landuse changes, crops or natural disasters, is of marginal importance in geological RS. Overcast or atmospheric haze may substantially depreciate or totally eliminate RS images from the analysis (again with the exception for radar images). All those issues are probably a reason why RS has been so far applied in geological mapping in rather rare and specific projects [3-5].

Hard rocks are exposed clearly essentially only on the hilltops, where they often form tors. On plains they are usually covered with residual sediments or regoliths, which in tropics may attain remarkable thickness up to a few hundred meters. Mineralogy and petrography of them are more or less closely related to the parent rocks. Therefore, spectral response of both is sometimes similar. Sediment displaced e.g. on a slope reveal spectral signature quite different to the parent rock and is unsuitable for RS analysis of the bedrock lithology. It applies even more evidently to the valleys and depressions sheeted by alluvium or prolluvium.

RS study aimed at both, lithological and structural mapping in Laos (Xiangkhouang and Vientiane provinces; Fig. 1), carried out at the Faculty of Geology, Geophysics and Environment Protection of the AGH-UST a few years ago (Projects contracted by the GeoExpert, unpublished), revealed that "classical" RS techniques (classification and band ratioing of multispectral images) supported with the analysis of DEM (Digital Elevation Model) and inspection of high resolution ortophotomaps (from repositories of the Google Earth, Bing etc.) may yield highly satisfactory results. Present paper is based on results and experience gained in this study.

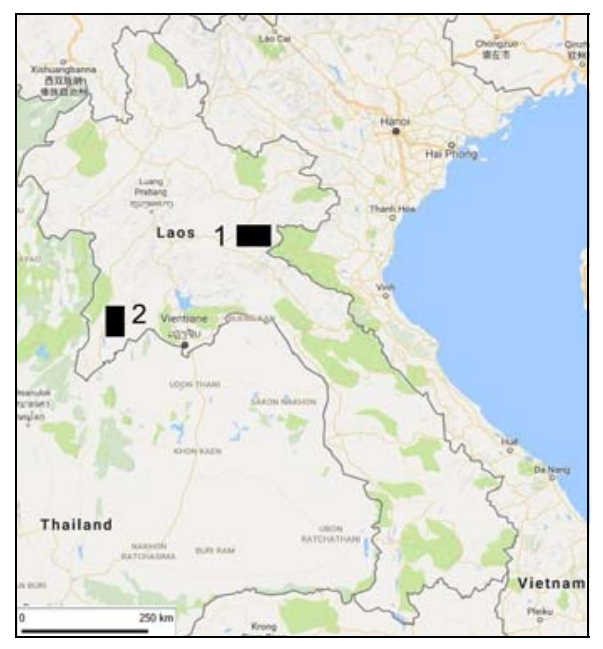

Fig. 1. Location of the study areas: 1 - Xiangkhouang, 2 - Vientiane (backdrop: Google Maps)

\section{Remotely sensed data}

The Landsat program (NASA) offers (free of charge) multitemporal images which have been acquired since July, 1972 [6]. This is the longest continuous satellite RS mission (carried out by three consecutive satellites and scanners). Sentinel 2 (European Space Agency) has been operating since June 2015 (Sentinel 2A) and March 2017 (identical Sentinel 2B). Images are freely available for ESA member states [7]. Both missions offer images of medium spatial resolution (10-60 m of pixel size) and good spectral inventory, comprising 6 to 12 bands. The bands range form visible blue/ultraviolet "blue-edge" to medium range infrared, plus thermal infrared and panchromatic. Particularly useful for lithological discrimination are bands around $2 \mu \mathrm{m}$ wavelength (bands 5 and 7 of TM and ETM+, band 6: SWIR-1 and 7: SWIR-2 of OLI, bands Mid IR1 and Mid IR2 of Sentinel 2). 
Radiometric resolution ranges from 8 bits (TM and ETM+) to 16 bits per pixel (OLI, Sentinel 2). This allows for quantification of the reflectance energy to, respectively, 256 and 65,536 levels. The latter figure seems, on one hand, incredible high, on the other, normally only a few thousands levels are "used" in practice.

New generation of commercial satellites and scanners World View 2 - World View 4 (Digital Globe) yields multispectral imagery of exceptionally good spatial resolution of a range of 1.24 (downsampled to $2 \mathrm{~m}$ for non US governmental use) to $3.7 \mathrm{~m}$, very good inventory of spectral bands - up to 16 (WV3) and good spectral resolution (11 bits per pixel). In addition they offer panchromatic images of $0.36-0.41 \mathrm{~m}$ pixel size (downsampled to $0.5 \mathrm{~m}$ ). WV satellites are relatively new. WV 1 operates since October 2009, while WV 2 from August 2014 [8]. Unfortunately, band of $\sim 2 \mu \mathrm{m}$ wavelength is missing from the WV2 scanner which imagery was used in the Vientiane study.

GDEM (Global Digital Elevation Model) data acquired by the ASTER satellite (NASA) were used for analysis of tectonic structures in the Vientiane study. Its spatial resolution declared to $30 \mathrm{~m}$ is in reality probably around $30-100 \mathrm{~m}$ and vertical accuracy is a few decimeters to dozen or so meters [9].

Finally, high resolution ortophotomap ( $\sim 0.5 \mathrm{~m}$ pixel size) acquired from the Google Satellite Maps repository was used for visual inspection and verification of the results of satellite data processing.

\section{Methods of processing and analysing of RS data}

Multispectral satellite RS images were processed and analyzed with rather standard methods applied normally in such a case. Good description of them can be found in, i.a., [1, 2]. In the present case they were:

- color composites created for general visual inspection and enhancement of selected objects (rock types) or phenomena (vegetation),

- band rtioing aimed at quantitative discrimination of selected objects or phenomena; vegetation index (NDVI) was used for creating vegetation mask applied later in classification,

- classification (unsupervised) aimed at producing lithological map.

GDEM was used for producing shaded relief map. It was examined in variable conditions of illumination: azimuth and angle of incident light.

Finally, results were verified by comparison to geological maps in scale 1:200,000 and $1: 1,000,000$.

\section{Results}

Spatial resolution (pixel size) of images is crucial for successful discrimination of small objects or objects exposed on small areas. The latter is the case of the tropical areas, masked by intensive and extensive vegetation. High resolution ortophotomap reveals very detailed image of the spot, while Landsat ETM+ image is quite general (Fig. 2).

Multispectral WV2 images give much better insight into the terrain than Landsat ETM+ or TM images (Fig. 3).

Spectral signatures of granitoids visible in gaps among trees and bush extracted from the WV2 images are much more different to signatures of vegetation obtained from the Landsat TM images (Fig. 4). Single pixel of the latter represents area of $30 \times 30 \mathrm{~m}$. It is very likely that both, rock and tree/bush might have been captured together. Therefore spectral signatures of rocks and vegetation are uniform. Well pronounced difference is visible only in band 4, smaller but probably significant occurs in band 3 . Small pixels of the WV2 images $(2 \times 2 \mathrm{~m})$ 
enable much more selective extraction of spectral signatures. They are significantly different in bands 6,7 and 8 , less pronounced in bands $2-5$. It is obvious that discrimination of rocks and vegetation is more effective on the WV2 imagery than on Landsat images. However, processing of the latter yields surprisingly good results on the terrain with less extensive vegetation or of bigger extent, where more general results are acceptable e.g., at the initial stage of prospecting (Fig. 7).
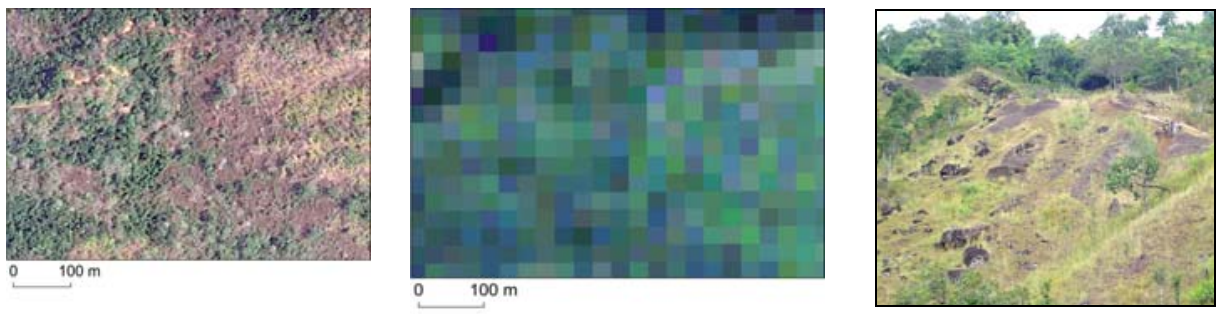

Fig. 2. Fragment of the Xiangkhouang study area shown on the ortophoto map (Google Maps; left), Landsat ETM+ "natural" colour composite RGB321 (centre) and ground photo (right)
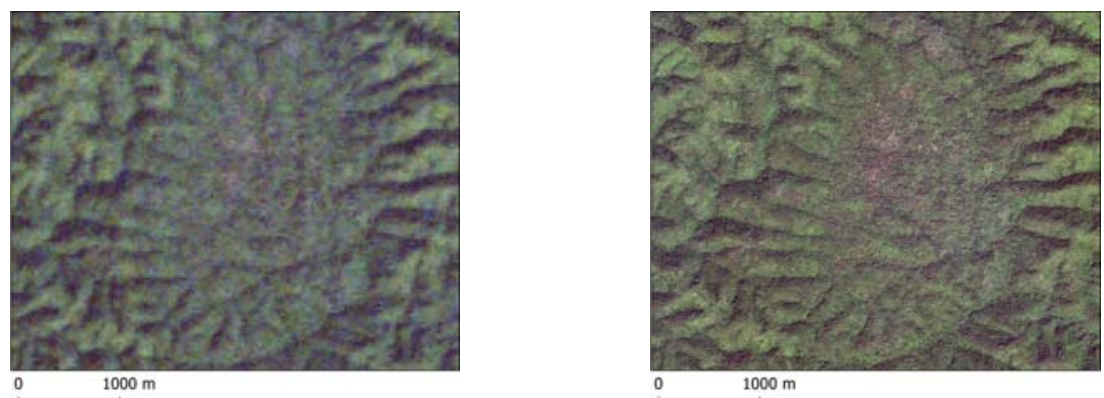

Fig. 3. Fragment of the Vientiane study area. "Natural" colour composites of Landsat TM RGB321 (left) and WV2 RGB532 (right). Intrusive Permo-Triassic granitoids form a dome. Barren rocks are represented by reddish-pink shades
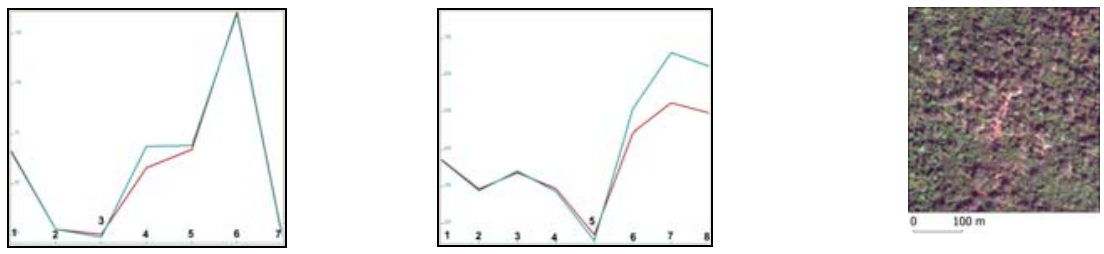

Fig. 4. Averaged spectral signatures (graphs) of granitoids (red lines) and vegetation (green lines) extracted from Landsat TM (left) and WV2 (centre) images. X - axis shows numbers of spectral bands numbers, $\mathrm{Y}-$ axis $-\mathrm{DN}$ values of reflectance.

Mixture of exposed rocks and vegetation (right) visible in close-up image of sampled area (WV2).

Vegetation which appears a particularly oppressive obstacle in scanning rocks and sediments in visible and infrared cannot be removed by any means of digital processing. However, vegetated areas can be eliminated prior to more advanced stages of the image processing to speed it considerably up. Vegetation mask, a binary (Boolean) raster map created by binarization of the vegetation index (e.g. NDVI) map, is a quite effective here. As one could 
expect, a share of not vegetated areas susceptible to lithological RS mapping may be quite small in tropics. In the presented fragment of the Vientiane area (Fig. 5) it is $\sim 19 \%$.
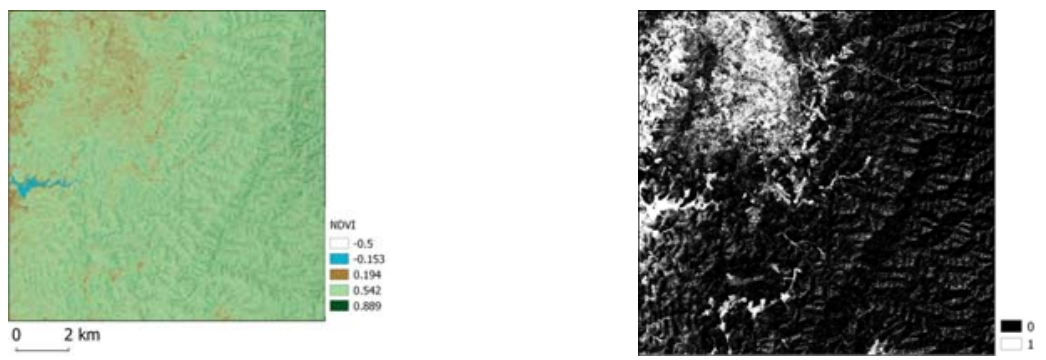

Fig. 5. Left: NDVI (Normalized Differential Vegetation Index). WV2 imagery. Vegetation is shown in shades of green, rocks, sediments and soil - in shades of brown, water -blue. Right: binary vegetation mask. 1 - areas with sparse or no vegetation, $0-$ vegetated areas. Threshold set to NDVI $=0.45$

\subsection{Band ratioing}

Clay, ferrous oxides, ferric iron, ferrous iron, laterite and gossan are standard band ratios applied commonly in geological mapping and prospection. They are calculated as a division of band in which reflectance of an object (e.g. iron oxides) is the highest by a band in which its reflectance is minimal. Atmospheric haze correction is sometimes added. Iron oxide ratio formula as applied to Landsat TM and ETM+ images is given below as an example:

$$
\text { (B3 - RMIN(,'All',I1)) / (B2 - RMIN(,'All',I2)) }
$$

where: B3 and B2 - band 3 (visible red) and 2 (visible green) of Landsat TM and ETM+ scanner; RMIN(,'All',I2) - component removing atmospheric haze (in C++ syntax).
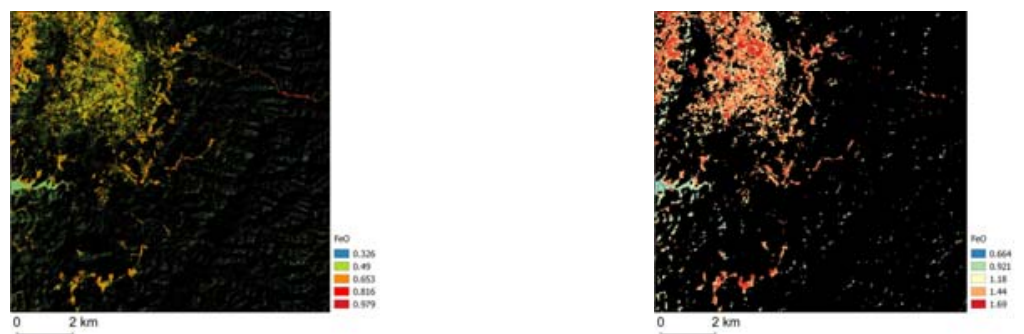

Fig. 6. Iron Oxide ratio masked with vegetation mask. Left: WV2 image, right: Landsat TM image. Relative values, not standardized

Band ratio is a quantitative measure of intensity of a phenomenon, e.g. oxidation of iron minerals in a rock and, therefore, indirectly, occurrence of the rock itself (Figs. 6 and 7). Scale of values is relative. It requires standardization in every particular case.

Image of band ratio of extensively vegetated area is more precise when high resolution WV2 images are used as an input (Fig. 6, left). Landsat images give more rough, generalized and less precise result (Fig. 6, right). However, processing of TM or ETM+ images gives satisfactory results in initial stages of prospection of bigger areas (Fig. 7). 


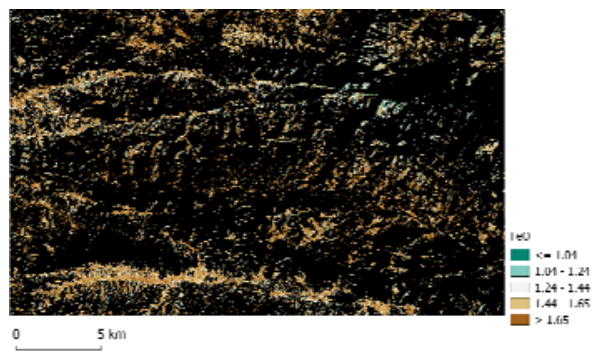

Fig. 7. Iron Oxide ratio masked with vegetation mask. Xiangkhouang area. Landsat ETM+ image. Relative values, not standardized

\subsection{Classification}

Classification is definitely the most advanced tool in the RS analysis. Unsupervised classification (ISODATA - Iterative Self-Organizing Data Analysis Technique) is conducted most frequently in areas poorly recognized that suffer from the lack of reference data (geological maps, field survey, sampling etc.). This is probably the case of most areas in tropics. All limitations of application of imagery of different scanners mentioned in previous sections apply here too. Two stages are new here:

1. pre-processing stage, i.e. selection and/or decorrelation of bands to be used in classification and

2. verification (qualitative or/and quantitative) of results of the classification.

Selection of spectral bands for classification may considerable improve its results. Normally short wave visible blue bands of WV2 scanner (Coastal Blue), Landsat OLI (CoastalAerosol) and Sentinel 2 (Blue Edge) are omitted. They are usually biased by atmospheric haze. Decorrelation of bands removes repeating information. Two methods are applied here: decorrelation stretch and extracting principal components (PC), usually first, second and third, sometimes fourth. Classification of PCs instead of original images may result in better separability of classes and their internal consistency. However, may considerable prolong the classification process to hours or even tens of hours (depending of the image size, number of PCs and efficiency of the computer).

Figure 8 shows result of unsupervised classification of WV2 multispectral images. Bands 2 thru 8 were used and 30 classes were extracted. Classes representing vegetation and built-up areas were eliminated.

Verification of the classification results requires reference data. The best of course is a field check, which is in most cases unfortunately unavailable. Geological maps are very good reference data, especially in big scales, i.e. 1:10.000 (very rare), 1:25.000 and 1:50.000. Medium and small scale maps (1:200.000 to 1:1.000.000) allow only for general reference. (BTW: areas mapped in 1:10.000 or 1:25.000 scale essentially do not require RS mapping!). Finally, high resolution ( $0.5 \mathrm{~m}$ or less of pixel size) ortophoto maps available for observation in e.g. Google Earth service or downloadable from Google Maps repository are good reference images. They allow observation of objects on the Earth's surface down to a size of a single stone or small outcrop in nearly natural colours. However, spectral analysis, although possible (after separation of RGB or HSI components), is not reliable. 

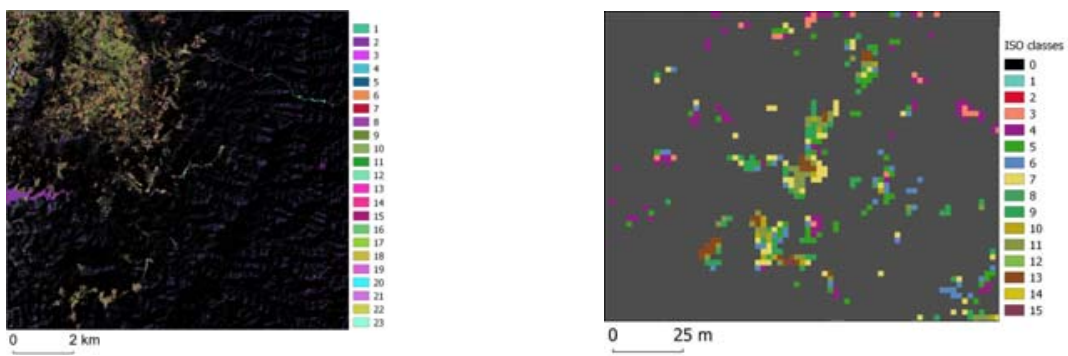

Fig. 8. Classified WV2 images masked with NDVI mask (left). Right: image zoomed and centred onto outcrops of granitoids shown in Figures 3 and 4. Barren rocks are represented on both maps by classes 9-12 shown in shades of green. Other classes represent probably rocks covered with weathering residuals and/or sparse vegetation.

Detailed geological map or results of the field mapping may be used as reference data for quantitative verification of the classified images. Cross tabulation (contingency table) is normally applied here (correlation testing cannot be conducted due to not random and not continuous character of the data). Statistical measures (overall accuracy, kappa statistics) allow for evaluation of classification results. If not satisfactory - the classification should be repeated with modified set of the data or/and with modified parameters.

\subsection{Analysis of DEM}

In many cases geological structure of both, hard rocks and sediments, reflects in geomorphology. Digital Elevation Model (DEM) which, further on, shows geomorphology, allows, therefore, for imaging and interpretation of geological structures. It applies to vegetated areas, including tropics, if DEM is acquired by active microwave (radar) scanning, as a large portion of microwave radiation penetrates vegetation. SRTM (Shuttle Radar Topographic Mission, NASA) and GDEM (Aster, NASA) are good sources of global DEM. Processing the latter appeared quite useful in structural interpretation of the Vientiane study area. Analysis of shaded relief model and DEM derivatives: slope angle, slope aspect, slope curvature, ruggedness, and microtopography was applied. Geological interpretation was supported by the Geological map of Cambodia, Laos and Vietnam [10]. Results are presented on a backdrop of shaded relief model (Fig. 9).
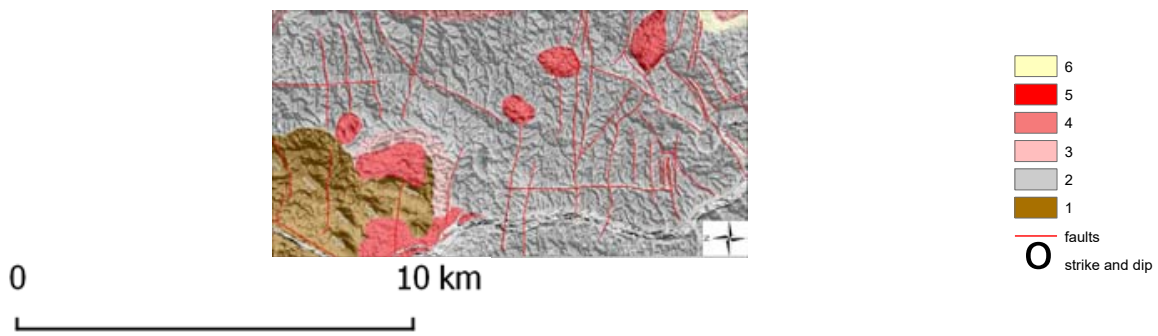

Fig. 9. Shaded DEM with interpreted lithology and structure. Illumination from NW. Items 1-6 are explained in the text

Analysis revealed 6 different types of the DEM textures.

1. Very coarse and massive texture with deep cuts and steep elevations, located in NW corner of the area shows probably thick bedded Devonian limestone and sedimentary 
rocks dipping generally towards NW. They form structurally controlled mountain ridges trending SW-NE.

2. Medium coarse and moderately sculptured texture which occupies the largest portion of the area. Probably represents Carboniferous limestone, mudstone, siltstone and other thick bedded sediments. The beds dip generally towards NW and SE and form probably tight SW-NE striking folds. Structurally controlled ridges run in SSW to SW-NNE to $\mathrm{NE}$ and $\mathrm{S}-\mathrm{N}$ directions.

3. Relatively medium grained texture that occurs in a crescent-shape along S, SE and E rim of the Devonian and intrusives (5). They may reflect Carboniferous and/or Devonian sediments changed by the intrusions. However, this texture may as well be a representation of subvulcanic intrusive rocks.

4. Fine to medium grained texture with low ridges and shallow valleys trending generally W-E represents probably Permian thin to medium bedded, relatively soft rocks. They occur along the E rim of the area and in its SE-most corner.

5. Medium to fine grained textures that stretch generally SE-NW across the area and border Devonian rocks in the NW corner. They reflect intrusive granitoids.

6. Nearly smooth texture of C-shape in SW corner of the area: Mekong river palaeo-valley.

Linear structures (faults) form 4 systems: W-E, N-S, SSW-NNE and SW-NE. Three latter seem dominating.

\section{Conclusions}

Intensive and extensive vegetation is the main factor controlling and limiting application of RS techniques in geological mapping of tropical regions. Therefore, good spatial resolution of imagery is crucial for detecting spectral response of rocks, sediments and soil "visible" essentially only in gaps among trees and bush and in clearings. The smaller pixel the more selective is the spectral signature gathered. Images of WV2 and WV3 scanners (Digital World) with $2 \mathrm{~m}$ pixel size represent actually top achievements in high resolution satellite RS imaging. Medium resolution Landsat TM, ETM+ and OLI as well as ESA Sentinel 2 scanners (10-60 m pixel size) seem not appropriate for analyzing extensively forested areas with small and rare clearings but may be good for mapping extensive areas where larger spots are dispersed with sparse or no vegetation (Fig. 6 right and 7).

Spectral and radiometric resolution are equally important in RS mapping in tropics and in any other area. Visible red and mid infrared wavelengths are important in mapping of lithology of rocks and sediments. In particular it applies to mid infrared bands around $2 \mu \mathrm{m}$ wavelength. "Blue-edge", visible blue and yellow (e.g. band 4 of WV2) ale less informative. Radiometric resolution of 11 and 16 bits per pixel seems actually a standard in modern scanners. It allows for very precise fragmentation of even very subtle characteristics of a reflectance spectrum of an object and, thus, discrimination of quite similar objects.

Thermal infrared (emissive) images represent quite another opportunity in many fields of RS processing and analysis, i.a. in lithological mapping. It was not applied in the presented study.

Digital Elevation Model can be used in very sophisticated analysis of geomorphology related to structures of bedrock and sediments. Laser scans (LIDAR) are suitable only for not vegetated areas. Highly penetrating microwave (radar) scanning yields very useful elevation data. Its relatively low resolution $(30-90 \mathrm{~m})$ is not a substantial obstacle in recognizing even small scale geological structures: faults, bedding and its dip and strike (and, further on, folds), intrusive bodies, abrupt changes of lithology, as well as covers of soft sediments.

Remote Sensing supplies us with almost perfect information on lithology and structure of rocks and sediments when applied in arid areas with no or sparse vegetation ( $\mathrm{N}$ and $\mathrm{E}$ Africa, Central Asia, W of both Americas, subpolar regions free of snow and ice). Used in tropical regions may yield interesting results, providing it is supplemented with interpolation 
of gained information to the vegetated ("hidden") fragments of the analyzed terrain. In this context we should ask here a question: is it possible to make any "classic" geological map without any interpolation of gathered observations and data over such "hidden" areas?

\section{Acknowledgements}

Publication resulted from the Faculty statute research project No. 11.11.140.320.

\section{References}

1. J.G. Liu, P.J. Mason, Image processing and GIS for Remote Sensing (Willey Blackwell, 2016)

2. R.P. Gupta, Remote Sensing Geology (Springer, 2003)

3. K. Won-In, P. Charusiri, Int. J. of Applied Earth Observ. and Geoinformation, 4, 183-193 (2003)

4. L.Q. Hung, O. Batelaan, IGARSS 2003, 4, 2964-2966 (2003)

5. B. Pradhan, S. Lee, Earth Science Frontiers, 14, 143-151 (2007)

6. https://landsat.usgs.gov

7. https://sentinels.copernicus.eu/web/sentinel

8. www.digitalglobe.com

9. https://terra.nasa.gov/about/terra-instruments/aster

10. Geological map of Cambodia, Laos and Vietnam, 1:1.000.000, Geol. Surv. of Vietnam, Hanoi (1991) 